\title{
2Hz-Electroacupuncture Attenuates Heroin-Seeking Behaviors via Adjusts CB1-Rs and CB2-Rs Expression in Relapse-Relevant Brain Regions of Heroin Self-Administration Rats
}

\author{
Lin CHEN ${ }^{1,2}$, Xiao-Kang GONG ${ }^{2}$, Chang-Long LENG ${ }^{2}$, Bao-Miao MA ${ }^{2}$, Qin RU ${ }^{2}$, Qi \\ $\mathrm{XIONG}^{2}$, Kai YUE ${ }^{2}$, Mei ZHOU ${ }^{2}$, Xiang TIAN ${ }^{2}$, Chao-Ying $\mathrm{LI}^{2}$, Yu-Xiang WU \\ ${ }^{1}$ School of Physical Education, Jianghan University, Wuhan, China, ${ }^{2}$ Wuhan Institutes of \\ Biomedical Sciences, Jianghan University, Wuhan, China
}

Received December, 2018

Accepted April 30, 2019

Epub Ahead of Print August 19, 2019

\section{Summary}

Opiate addiction has a high rate of relapse. The accumulating evidence shows that electroacupuncture (EA) may be effective for the treatment of opiate relapse. However, the change of expression of CB1-Rs and $\mathrm{CB} 2-\mathrm{Rs}$ involve in $2 \mathrm{~Hz}$ EA anti-relapse pathway is still unclear. To explore the changes of expression of CB1-Rs and CB2-Rs, heroin self-administration (SA) model rats were adopted and treated using $2 \mathrm{~Hz} E A$. The expressions of CB1-Rs and CB2-Rs were observed using immunohistochemistry method. The results showed that, compared with the control group, active pokes in the heroin-addicted group increased, while the active pokes decreased significantly in $2 \mathrm{~Hz}$ EA group compared with heroin-addicted group. Correspondingly, the expression of CB1-Rs in prefrontal cortex (PFC), hippocampus (Hip), nucleus accumbens (NAC) and ventral tegmental area (VTA) all increased significantly while the expression of CB2-Rs in those relapse-relevant brain regions decreased obviously in heroin-addicted group when compared with the control group. In addition, the expression of CB1-Rs obviously decreased in the $2 \mathrm{~Hz}$ EA group while the expression of $\mathrm{CB} 2-\mathrm{Rs}$ in those relapserelevant brain regions increased significantly when compared with the heroin-addicted group. It indicated that $2 \mathrm{~Hz}$ EA could attenuate the heroin-evoked seeking behaviors effectively. The anti-relapse effects of $2 \mathrm{~Hz}$ EA might be related to the decrease of CB1-Rs and increase of CB2-Rs expression in relapse-relevant brain regions of heroin $\mathrm{SA}$ rats.
\end{abstract}

\section{Key words}

Heroin self-administration • 2Hz-electroacupuncture • Heroinseeking behaviors $\bullet$ CB1-Rs and CB2-Rs

\section{Corresponding author}

Y.-X. Wu, School of Physical Education, Jianghan University, Wuhan 430056, China. Fax: +86-27-84261507. E-mail: hdycl@126.com

\section{Introduction}

A high rate of relapse in drug abuse (such as heroin) during abstinence is defined as a feature of drug addiction (Dong et al. 2017). The ultimate aim in the treatment of heroin abuse is the abstinence and prevention of heroin relapse (Kosten et al. 2003). So far, the prevention of heroin relapse is still an urgent medical and social problem, especially in China (Tang et al. 2007).

Electroacupuncture (EA) is a modification of this technique which small electrical currents were applied to acupoint though needle and appeared to have more effective effects in many specific clinical and research settings (Zhao et al. 2008). EA-induced analgesic effect is used widely to alleviate pain (Chen et al. 2009, Da Silva et al. 2015, Gelbier et al. 2016, Han et al. 2004). EA were applied with great success to attenuate behavior of morphine withdrawal in animals and addicts since 1972 (Cui et al. 2013, Shen et al. 2014, Tseung et al. 1974).

The endocannabinoid system which consisted of the endogenous cannabinoids and cannabinoid receptors also plays an influential role in the mesolimbic dopamine (DA) system and drug addiction (Sagheddu et al. 2015, 
Wills et al. 2016). Recent studies reported that the discovery and functional presence of CB1-Rs (Mitrirattanakul et al. 2007) and CB2-Rs (Onaivi et al. 2006, Onaivi et al. 2011) in mammalian brain might be crucial in depression and drug abuse. The mesolimbic dopamine system is thought to play a major role in the reinforcing effects of drugs abuse, especially in prefrontal cortex (PFC), hippocampus (Hip), nucleus accumbens (NAc) and ventral tegmental area (VTA) (Koob et al. 1992, Nestler et al. 2004). Some studies reported that the up-regulation of hippocampal CB1-Rs may contribute to the increasing alcohol consumption (Mitrirattanakul et al. 2007) and the brain cannabinoid CB2-Rs modulated cocaine's addictions in mice probably by a dopaminedependent pathway (Morales et al. 2012, Xi et al. 2011).

Based on the studies above and our previous finding that $2 \mathrm{HzEA}$ could significantly increase the anandamide level in inflammatory pain tissues (Chen et al. 2009) and up-regulate the local CB2-Rs expression (Zhang et al. 2010), we hypothesized that the $2 \mathrm{~Hz}$ EA could effectively inhibit the heroin addiction behaviors via regulating the expression of $\mathrm{CB} 1-\mathrm{Rs}$ and $\mathrm{CB} 2-\mathrm{Rs}$ in relapse-relevant brain regions of heroin $\mathrm{SA}$ rats. To explore the change of expression of CB1-Rs and CB2-Rs, we detected the inhibitory effect of $2 \mathrm{~Hz}$ EA on heroin priming seeking behavior in SA rats through an extinction/reinstatement protocol. Meanwhile, the expressions of CB1-Rs and $\mathrm{CB} 2-\mathrm{Rs}$ in the relapserelevant brain regions were assessed with immunohistochemistry method.

\section{Materials and Methods}

Animals

Male Sprague-Dawley rats, weighing 280-300 g at the beginning of the experiments, were purchased from Vital River Laboratory Animal Technology Co., Ltd, Beijing, China. The animals were maintained under a $12 \mathrm{~h}$ reversed light/dark cycle (with darkness starting from 8:00 am) with controlled room temperature and humidity. Tap water was made available ad libitum and food was restricted to $20 \mathrm{~g}$ per day. All procedures were performed in accordance with the National Institutes of Health Guide for the Care and Use of Laboratory Animals.

\section{Surgery and experimental apparatus}

Procedures of surgery and experimental apparatus followed our previous study (Yue et al. 2012).
Briefly, after 1 week of acclimation, a permanent intravenous catheter was surgically implanted and secured to the right jugular vein under deep anesthesia. Following surgery, each rat was housed and was allowed at least 7 days of recovery during which they received a daily intravenous infusion of gentamicin $(0.16 \mathrm{mg} / \mathrm{kg})$ and heparinized ( $1 \%$ ) sterile saline solution to keep the catheter clean and unobstructed. Heroin SA was carried out in operant chambers which encased in sound- and light-attenuating cubicles and fans that provided ventilation (Anilab Software \& Instruments Co., Ltd., China) (Wang et al. 2010). The front panel was armed with two nose-poke operandi (ENV-114M, Med Associates, USA) located $9 \mathrm{~cm}$ above the floor of the chambers. A red stimulus light was placed in each nosepoke and a white house light was fixed on the opposite wall. A single active nose poke resulted in a $5 \mathrm{~s}$ heroin infusion meanwhile the pump is on. Concurrently, the red cue light in this nose-poke was on for $5 \mathrm{~s}$ while the white house light was turned off. A $20 \mathrm{~s}$ timeout period was then allowed, during which the red cue light was turned off and further nose pokes had no additional consequence, but were recorded. After the timeout period, the white house light was once again turned on. Inactive nose pokes were also recorded. Each SA session started when the white house light was turned on.

\section{Heroin self-administration training (acquisition, maintenance and reinforcement)}

Heroin self-administration training was developed with fixed ratio program. In short, after recovered, rats were allowed to self-administer heroin $(30 \mu \mathrm{g} / \mathrm{kg}$ per inf) under an fixed-ratio 1 (FR1) schedule of acquisition and maintenance and to nose-poke for $3 \mathrm{~h}$ each day. The acquisition sessions were carried out until stable heroin intake was reached (typically within 12-14 days). Responding was considered stable when animals displayed accurate discrimination between the active and the inactive nose-poke, with the day-to-day difference in the number of active nose pokes of less than $15 \%$ for 3 consecutive days. Rats not satisfying the acquisition criterion were excluded from the experiment. The same experimental procedures were used for the rats of the control group $(n=8)$ except the heroin was substituted with the same volume of saline. Twenty-four rats developed a stable pattern of heroin intake. Thereafter, these rats were randomly divided into three groups ( $n=8$ per group), that is heroin-addicted group, sham EA group and $2 \mathrm{~Hz}$ EA group. The rats of $2 \mathrm{~Hz}$ EA 
group were treated with $2 \mathrm{~Hz}$ EA stimulus $(1 \mathrm{~mA}$ and $0.1 \mathrm{~ms}$ pulse width) for $30 \mathrm{~min}$ on acupoints zusanli (ST36) and sanyinjiao (SP6) $30 \mathrm{~min}$ before the experimental sessions according to the methods reported in the existing literature (Han et al. 1993). The same experimental procedures were used for the rats of sham EA group without electrical stimulus. The same experimental procedures were used for the rats of control and heroin-addicted group without $2 \mathrm{~Hz}$ EA stimulus. The reinforcement effects (numbers of inactive/active nose poke on an FR 1 schedule) were recorded at the last day of the training. Rats were then allowed to training return to their basal rate of responding to heroin before being switched to the subsequent extinction phase.

\section{Extinction and sham $/ 2 \mathrm{~Hz}$ EA treatment}

Those rats were abstinent from heroin for two weeks, during which they stayed in the operant chambers for $2 \mathrm{~h}$ per day without cue light, house light, sound of pump and heroin infusion. Extinguished behavior, in most cases, took place at around day 10-12. Previous study found that treatment with acupuncture at ST36 or SP6 during the withdrawal period inhibited alcohol withdrawal syndrome of rats undergoing ethanol injection (Kim et al. 2005), so the rats of sham $/ 2 \mathrm{~Hz}$ EA groups continued to be treated by sham or $2 \mathrm{~Hz}$ EA stimulus ( $1 \mathrm{~mA}$ and $0.1 \mathrm{~ms}$ pulse width) for $30 \mathrm{~min}$ on acupoints zusanli (ST36) and sanyinjiao (SP6) for every day before the extinction procedure according to the methods reported in the existing literature (Han et al. 1993) in our present study. The same experimental procedures were used for the rats of control and heroin-addicted group without EA stimulus.

\section{Reinstatement}

After extinction, heroin-induced reinstatement was examined. During testing, the rats were reintroduced into the operant cages for $2 \mathrm{~h}$ training. Before the beginning of the training, the rats were administrated with heroin priming (subcutaneous injection of heroin at $0.25 \mathrm{mg} / \mathrm{kg}$ ). A single active nose-poke obtained with a programmed consequences but no heroin injection. The numbers of active nose-poke and inactive nose-poke were both recorded.

\section{Locomotor testing}

To further characterize the sedative properties of sham EA and $2 \mathrm{~Hz}$ EA, twenty-four heroin-free rats were tested for the effects of EA on locomotor activity. Rats were tested for their locomotor responses using an automated photocell system (Anilab Software \& Instruments Co., Ltd., China) consisting of eight identical black Plexiglas chambers $(43 \times 43 \times 35 \mathrm{~cm})$ in light- and sound-controlled cubicles (Cheng et al. 2011). Each chamber was equipped with a video camera on the top, which was interfaced with a computer to record the movement of the rats in the chambers. The locomotor activity of each rat was analyzed by employing an AniLab ver 4.3 analysis software package (Anilab Software \& Instruments Co., Ltd., China) (Zhang et al. 2006) and was expressed as the total distance traveled (in millimeters) during a 2-h period that began 30 min after sham EA or $2 \mathrm{~Hz}$ EA administration.

\section{Immunohistochemically staining}

Immediately after behavioral testing, the expression of CB2-Rs in prefrontal cortex (PFC), hippocampus (Hip), nucleus accumbens (NAc) and ventral tegmental area (VTA) were investigated. The rats were systemically perfused with $4 \%$ paraformaldehyde during 2-3\% isoflurane-induced anesthesia. The brain were then isolated post-fixed overnight in $4 \%$ paraformaldehyde in PBS. After paraffin embedding, brain paraffin tissue blocks were cut into $4 \mu \mathrm{m}$-thick slices. After dewaxing and rehydration treatment, microwave antigen retrieving and endogenous peroxidase blocking, slices were then incubated with rabbit anti-CB1-Rs (1:200, Cayman, USA) and rabbit anti-CB2-Rs (1:200, Cayman, USA) diluted in PBS for overnight at $4{ }^{\circ} \mathrm{C}$ separately. After 3 rinses in PBS, the sections were then incubated with the secondary antibody (HRP conjugated goat anti-rabbit, 1:1,000, Abcam, Cambridge, UK) at $37^{\circ} \mathrm{C}$ for $40 \mathrm{~min}$. After three washes again in PBS, slices were mounted in glycerol and covered. The sections were examined with microscopy (Olympus BX51, Japan) and taking photographs. Optical densities of CB1-Rs and CB2-Rs were counted in a blinded fashion on 12-16 randomly selected brain sections from 8 animals per each condition. The results were expressed as relative optical density from these sections. Negative controls in which PBS was used instead of the primary antibody were processed in the same manner.

\section{Statistical analysis}

All results were expressed by mean \pm S.E.M. statistical significance of results was analyzed with oneway or two-way ANOVA. All statistical analyses were performed by utilizing SPSS for Windows, version 11.5 
(SPSS Inc., Chicago, IL, USA). $\mathrm{p}<0.05$ was considered to be statistically significant.

\section{Results}

\section{Effects of $2 \mathrm{~Hz}$ EA on heroin reinforcement}

Heroin reinforcement effect refers to the coercive behavior of pursuing heroin without self-restraint, which reflects the degree of heroin craving. After a stable pattern of heroin intake was developed in rats, the heroin SA reinforcement effects of these four groups were tested (sham EA or $2 \mathrm{~Hz}$ EA treatment performed $30 \mathrm{~min}$ before the experimental session). The effects on heroin SA reinforcement of rats on an FR 1 schedule were shown in Figure 1. Significant differences $\left(F_{3,51}=28.36, p<0.01\right)$ were observed (presented as the number of active nose pokes) among control group and other three heroin-treated groups which indicated the heroin SA model was successfully built. The number of active nose pokes of $2 \mathrm{~Hz}$ EA group significantly decreased compared with that in the heroin-addicted group $\left(\mathrm{F}_{3,51}=32.87, \mathrm{p}<0.01\right)$. Comparisons also revealed a significant difference between $2 \mathrm{~Hz}$ EA groups and the sham EA group $\left(\mathrm{F}_{3,51}=32.87, \mathrm{p}<0.01\right)$. No significant differences were observed in the number of inactive nose pokes $\left(\mathrm{F}_{3,51}=200.36, \mathrm{p}>0.05\right)$. These results indicated that $2 \mathrm{~Hz}$ EA could effectively inhibit the heroin reinforcement.

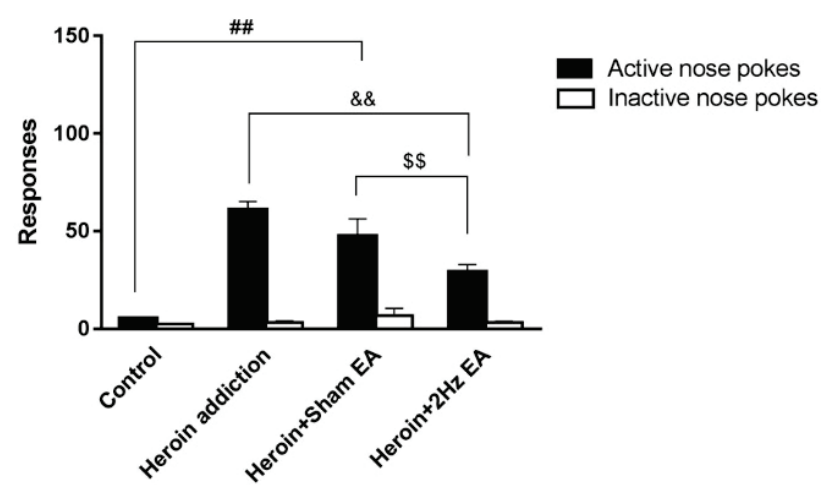

Fig. 1. $2 \mathrm{~Hz}$ EA stimulation attenuated heroin self-administration reinforcement in rats. Values are given in mean \pm SEM of eight animals per group. ${ }^{\# \#} \mathrm{p}<0.01$, as compared with control group. \&\& $p<0.01$, as compared with heroin-addicted group. ${ }^{\$ \$} p<0.01$, as compared with sham EA group.

\section{Effects of $2 \mathrm{~Hz}$ EA on heroin-induced reinstatement}

Our result showed that the effect of $2 \mathrm{~Hz}$ EA on heroin-induced reinstatement (Fig. 2). The number of active nose-pokes significantly increased in heroin treated groups when compared to the control group $\left(\mathrm{F}_{3,56}=5.566\right.$, $\mathrm{p}<0.01)$. And the number of active nose-pokes significantly decreased in $2 \mathrm{~Hz}$ EA group as compared with heroin-addicted group $\left(\mathrm{F}_{3,56}=8.362, \mathrm{p}<0.01\right)$. There was a significant difference between sham EA group and the heroin-addicted group in the number of active nose pokes $\left(\mathrm{F}_{3,56}=8.362, \mathrm{p}<0.05\right)$. By contrast, no significant differences were observed in the number of inactive nosepokes during reinstatement group $\left(\mathrm{F}_{3,56}=32.1, \mathrm{p}>0.05\right)$, suggesting that rats retained a good discrimination between the active nose-poke and inactive nose-poke. It was showed that $2 \mathrm{~Hz}$ EA stimulation could significantly reduce the heroin-induced reinstatement behavior in heroin-addicted rat.

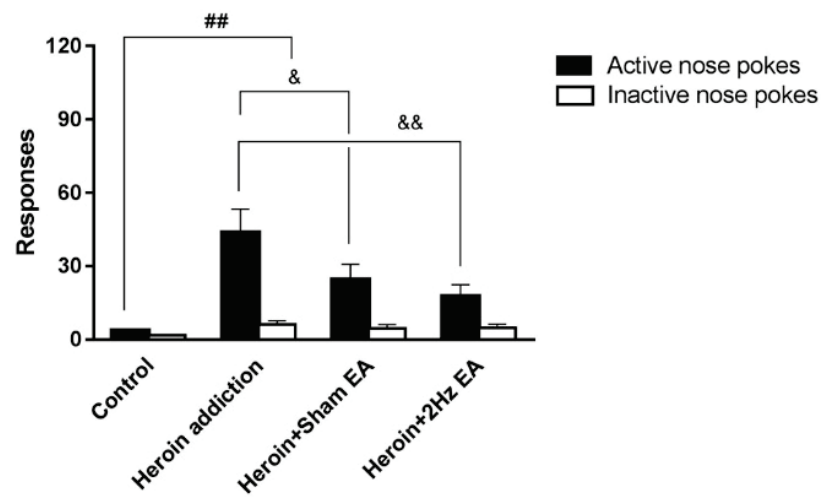

Fig. 2. $2 \mathrm{~Hz}$ EA stimulation attenuated heroin-induced reinstatement in rats. Values are given in mean \pm SEM of eight animals per group. ${ }^{\# \#} \mathrm{p}<0.01$, as compared with control group; \&\& $p<0.01$, as compared with heroin-addicted group; ${ }^{*} p<0.05$, as compared with heroin-addicted group.

\section{Effects of $2 \mathrm{~Hz}$ EA on locomotor activity}

To further examine the sedative properties of $2 \mathrm{~Hz}$ EA, the effects of heroin, sham EA and $2 \mathrm{~Hz}$ EA on locomotors activity (total distance traveled $/ 2 \mathrm{~h}$ ) on normal rats were examined as shown in Figure $3(n=5)$. Heroin treatment could reduce the locomotors activity of rats significantly $(p<0.05)$. The results showed that there was no significant reduce in locomotor activity in these two EA-treated groups compared to the control group $(p>0.05)$. It was indicated that EA-therapy itself had no ataractic influence on heroin self-administration reinforcement or heroin-induced reinstatement.

Effects of $2 \mathrm{~Hz} \mathrm{EA}$ on CB1-Rs and CB2-Rs Protein expression in PFC, Hip, NAc and VTA of heroin SA rats

Immunohistochemical technique is beneficial to the localization and semi-quantification of the receptor at the cellular level. We applied this technique to explore the differences of CB1-Rs and CB2-Rs expressions in the 
brain regions related to relapse. The present results showed that the expression of CB1-Rs in PFC, Hip, NAC and VTA of brain areas significantly increased in heroinaddicted group when compared to the control group $(p<0.05)$, but decreased significantly in $2 \mathrm{~Hz}$ EA group as compared with heroin-addicted group $(\mathrm{p}<0.01)$ (Fig. 4). Interestingly, the expression of CB2-Rs in PFC, Hip, $N A C$ and $V T A$ of brain areas significantly reduced in heroin-addicted group when compared to the control group ( $p<0.05$ ), but enhanced significantly in $2 \mathrm{~Hz}$ EA group as compared with heroin-addicted group $(\mathrm{p}<0.01)$ (Fig. 5). These results indicated that $2 \mathrm{~Hz}$ EA stimulation could significantly attenuate the expression of CB1-Rs and enhance the expression of CB2-Rs in addictionrelative brain areas in heroin-addicted rats.

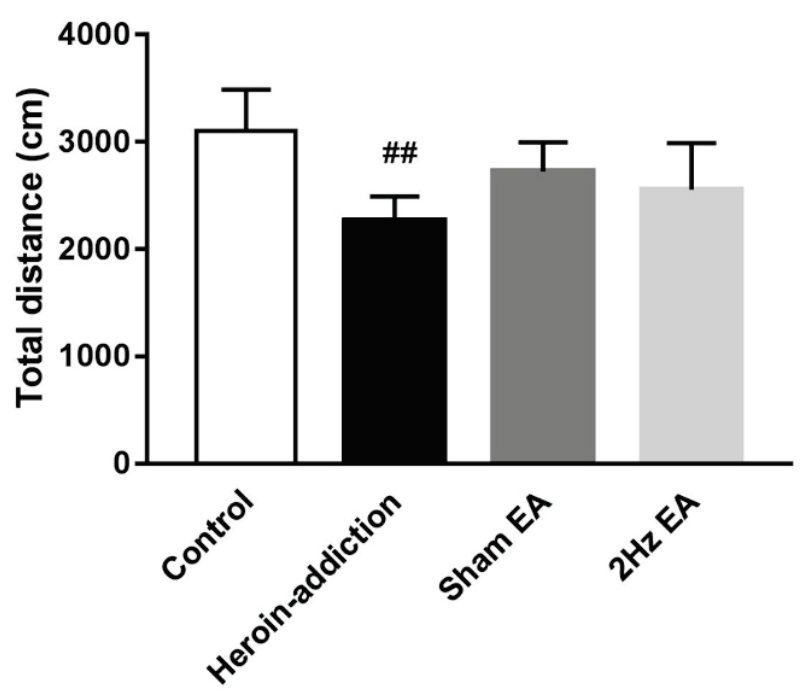

Fig. 3. $2 \mathrm{~Hz}$ EA stimulation did not affect the locomotor activity on normal rats. Values are given in mean \pm SEM of eight animals per group. ${ }^{\# \#} \mathrm{p}<0.01$, as compared with control group.

\section{Discussion}

Previous animal studies found that the underlying neurobiological mechanisms of EA preventing opiate withdrawal syndrome and relapse might be mediated by $\mu$ and $\delta$-opioid receptors and probably via accelerating the synthesis and release of enkephalin in the NAc (Cui et al. 2013, Han et al. 1993, Liang et al. 2010, Shi et al. 2003, Wu et al. 1999). Our present study firstly reported that the $2 \mathrm{~Hz}$ EA could effectively inhibit the heroin addiction behaviors which induced by heroin priming via regulating the expression of CB1-Rs and CB2-Rs in relapse-relevant brain regions of heroin $\mathrm{SA}$ rats.

Accumulated animal studies adopt the CPP rat model to study the effectiveness of EA. Compared with other models of addiction, the heroin SA model is widely used to study the neurobiological mechanisms involved in drug addiction (Garcia Pardo et al. 2017) because of the great similarity between the results obtained from the animal model and those human addictive behaviors (Mead et al. 2014). Both our and other related studies also showed that $2 \mathrm{~Hz}$ EA could effectively prevent the reinstatement to heroin seeking elicited by conditional cue in the heroin SA models (Chen et al. 2014, Liu et al. 2012, Zachariou et al. 2006). Here, we continued to adopt the heroin SA rat model to observe the influence of $2 \mathrm{~Hz}$ EA on seeking-behaviors which induced by heroin priming and to explore the potential mechanism. A significantly difference was observed in the number of active nose pokes (represent the heroin selfadministration) among control group and heroin-treated group which suggested that the heroin SA model was successfully built in our present study.

The withdrawal syndrome in morphinedependent rats could be effectively suppressed by $100-\mathrm{Hz}$ EA (Han et al. 1993, Wu et al. 1999). Morphine-induced CPP could be successfully suppressed by $2-$ or $100-\mathrm{Hz}$ EA (Liang et al. 2010, Shi et al. 2003). Our present results showed that both heroin SA reinforcement and reinstatement (represents heroin seeking and relapse behaviors, showed in active nose pokes) induced by heroin priming in $2 \mathrm{~Hz}$ EA group decreased significantly when compared with the heroin-addiction group. Meanwhile, there was no significant reduction of locomotor activity in 2HzEA-treated group when compared with control group. It indicated that $2 \mathrm{~Hz}$ EA could effectively inhibit the reinforcement and reinstatement of heroin self-administration rats and the 2Hz EA-therapy itself had no ataractic influence on heroin-seeking and relapse behaviors.

Generally speaking, the inhibitory effects of $2 \mathrm{~Hz}$ EA on the expression of the morphine CPP might be mediated by $\mu$ - and $\delta$-opioid receptors, possibly via accelerating both the release and synthesis of enkephalin in the brain (Liang et al. 2010). Nowadays, the mesolimbic dopamine system is thought to play a major role in the reinforcing effects of drug abuse, especially in PFC, Hip, NAc and VTA (Koob et al. 1992, Nestler et al. 2004). Study found that acupuncture significantly decreased dopamine release and behavioral hyperactivity which induced by a systemic morphine challenge but the detailed mechanism involved in it has not been fully investigated (Kim et al. 2005). 
A
PFC

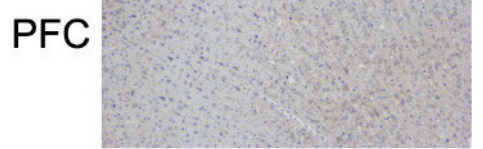

Heroin-addiction Heroin+Sham EA Heroin $+2 \mathrm{~Hz}$ EA
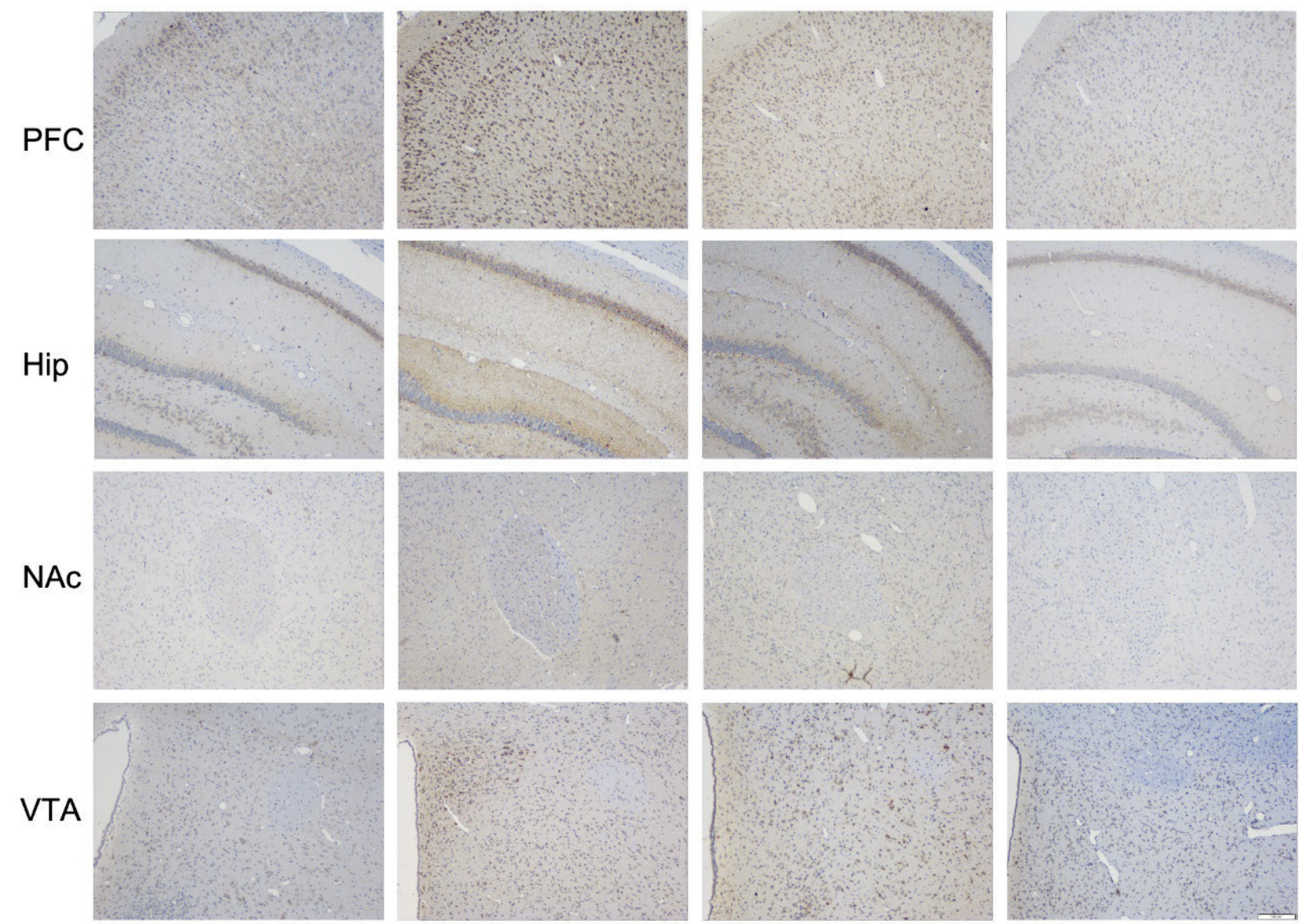

B
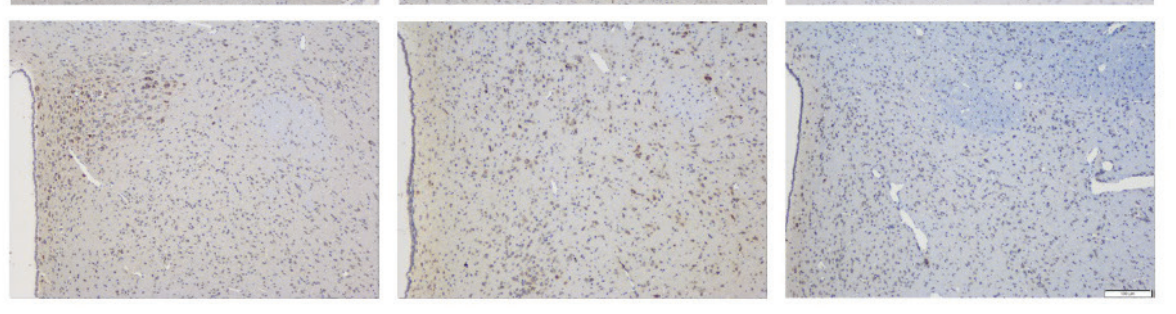

\section{CB1R Expression}

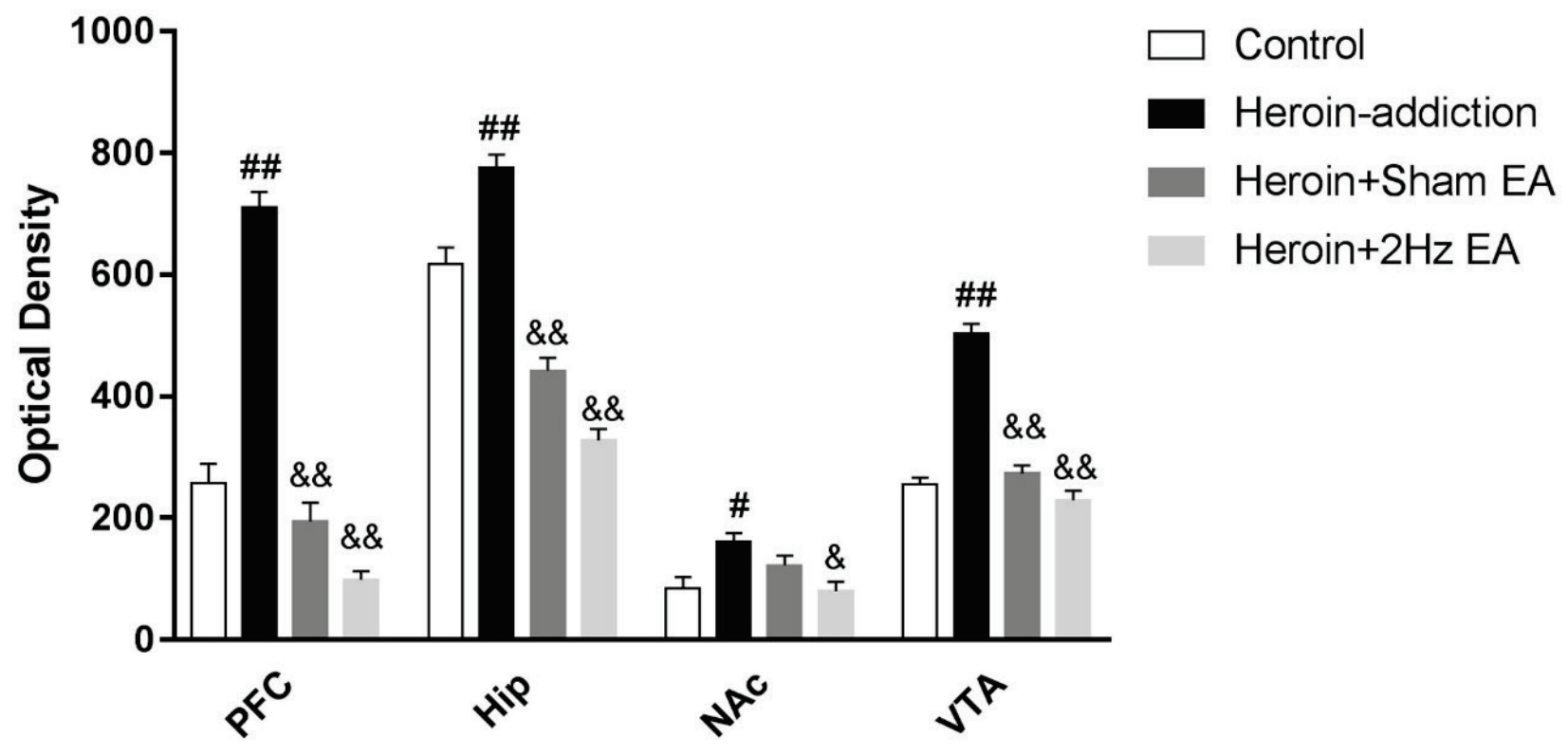

Fig. 4. The different expressions of CB1-Rs in PFC, Hip, NAC and VTA among control group, heroin-addicted group, Sham EA and $2 \mathrm{~Hz}$ EA group. (A) Representative immunohistochemical images of CB1 receptor in different brain regions; (B) Histogram showing the differences of CB1 receptor expression in different brain regions. Values are given in mean \pm SEM of eight animals per group. ${ }^{*} p<0.05$, ${ }_{\# \#} \mathrm{p}<0.01$, as compared with control group; ${ }^{\&} \mathrm{p}<0.05$, \&\& $\mathrm{p}<0.01$, as compared with heroin-addicted group. Scale bar, $100 \mu \mathrm{m}$. 


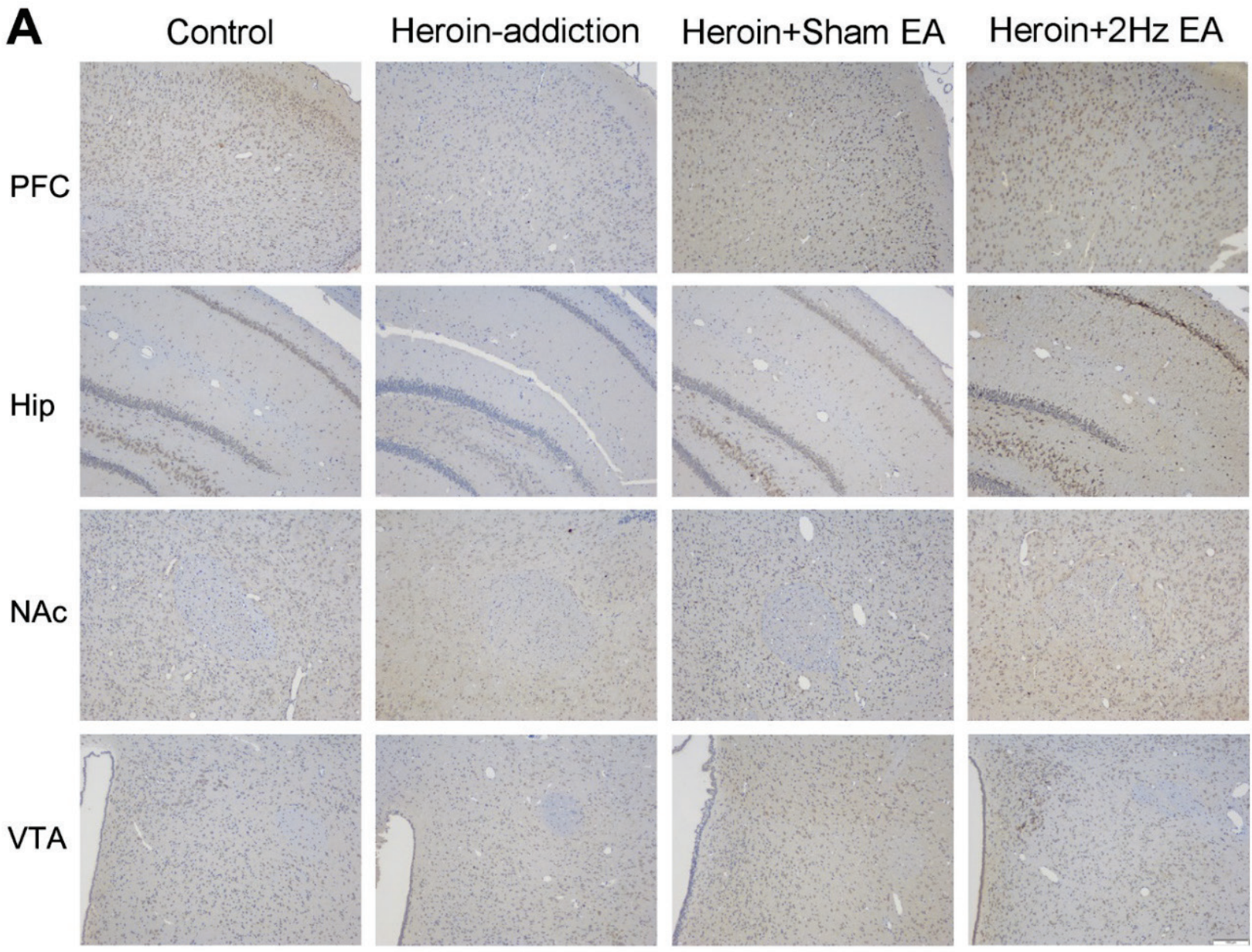

B

\section{CB2R Expression}

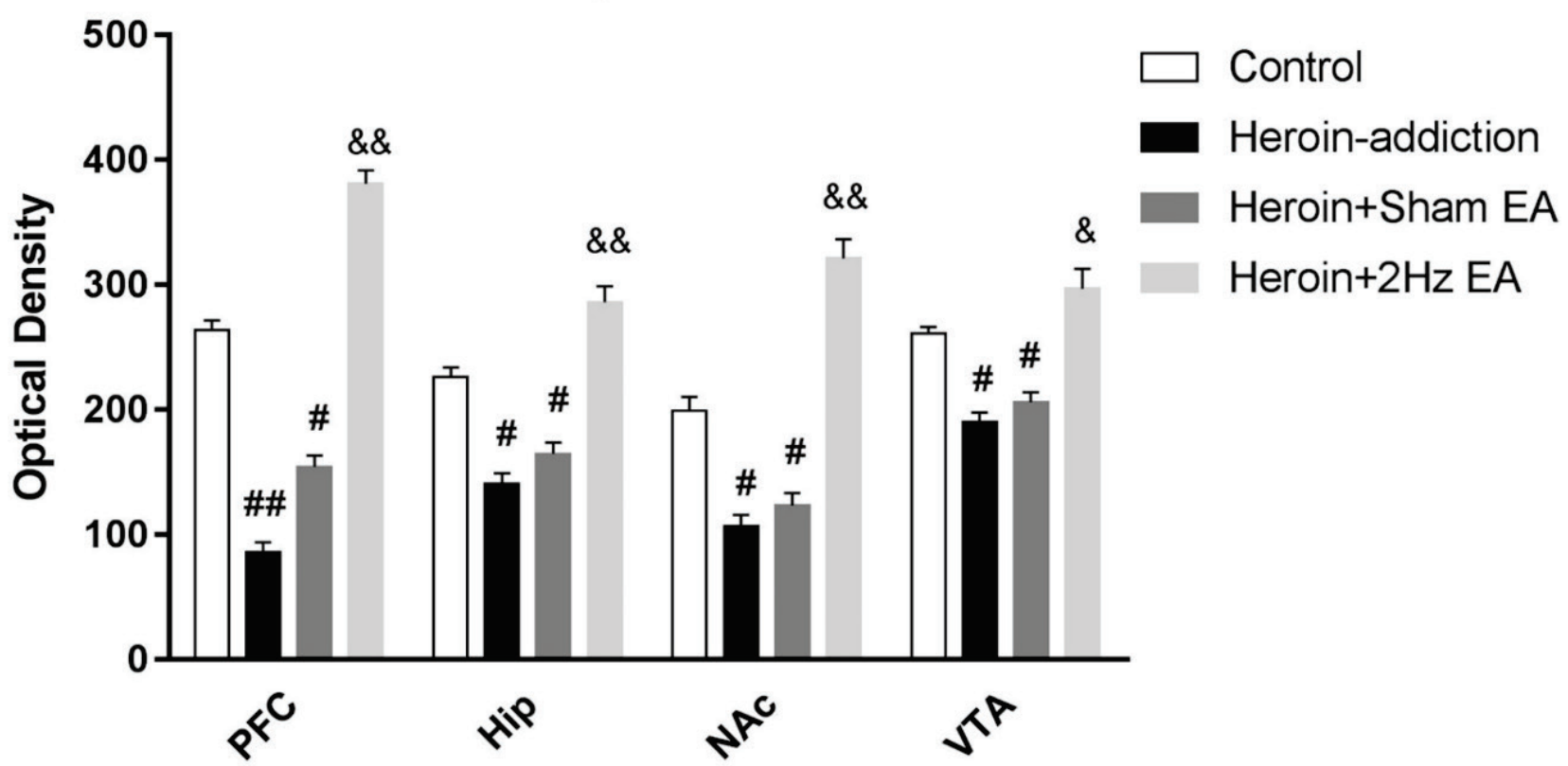

Fig. 5. The different expressions of CB2-Rs in PFC, Hip, NAC and VTA among control group, heroin-addicted group, Sham EA and $2 \mathrm{~Hz}$ EA group. (A) Representative immunohistochemical images of $\mathrm{CB} 2$ receptor in different brain regions; (B) Histogram showing the differences of CB2 receptor expression in different brain regions. Values are given in mean \pm SEM of eight animals per group. ${ }^{*} p<0.05$, $\# \#<<0.01$, as compared with control group; ${ }^{\&} p<0.05,{ }^{\text {\& }} \mathrm{p}<<0.01$, as compared with heroin-addicted group. Scale bar, $100 \mu \mathrm{m}$. 
Recently, it was proved that drugs, which enhanced brain reward, had common actions on the DA reward system and on animal behaviors closely relates to endocannabinoid system's function (Gardner et al. 2005). Studies reported that the endocannabinoid system also involved in modulating animal drug-seeking behaviors (Chen et al. 2017, Xi et al. 2011). The presence of CB1-Rs throughout brain reward circuits and the rewarding effects produced by $\mathrm{CB} 1-\mathrm{Rs}$ activation allows for the possible influence on the rewarding effects produced by non-cannabinoid substances. In general, drugs activate CB1-Rs appear to facilitate the rewarding effects of non-cannabinoid drugs (Parsons et al. 2015). CB1-Rs agonists increase the motivational and reinforcing effects of alcohol, nicotine and opiates indexed by animal models of drug reward while CB1-Rs antagonism diminished CB1-Rs signaling (either genetic deletion or pharmacological antagonism) attenuates the motivational and rewarding effects of these drugs (Panagis et al. 2014, Serrano et al. 2011). A low density of CB2-Rs expression was found in mesolimbic DA neurons, which might modulates cocaine's addictions in mice probably by a dopamine-dependent mechanism (Morales et al. 2012, Xi et al. 2011). Activation of CB2-Rs (Gi/o coupled receptor (Bayewitch et al. 1995)) on DA neurons in the midbrain VTA could directly inhibit dopaminergic neurons and decrease NAc DA release (Bayewitch et al. 1995, Xi et al. 2011). Therefore, the CB1-Rs and CB2-Rs involved in the mesolimbic dopamine system (such as PFC, Hip, NAc and VTA) might play important roles in heroin addiction. Our results demonstrated that the expression of CB1-Rs significantly increased while the expression of CB2-Rs decreased significantly in PFC, Hip, NAc and VTA in heroin SA rats when compared with those control rats.

Our previous study showed that $\mathrm{CB} 2$ receptors contribute to the analgesic effect of $2 \mathrm{~Hz}$ EA in inflammatory pain. $2 \mathrm{~Hz} \mathrm{EA}$ could significantly upregulate the local CB2-Rs expression (Zhang et al. 2010). In addition, study reported that the endocannabinoid system might be a primary mediator and regulatory factor of acupuncture's beneficial effects in drug addiction ( $\mathrm{Hu}$ et al. 2017). We hypothesized that $2 \mathrm{~Hz}$ EA could down-regulate the expression of CB1-Rs and up-regulate the expression of CB2-Rs in relapserelevant brain regions and help attenuating the heroin seeking behaviors. Our results demonstrated that the expression of CB1-Rs significantly decreased while the expressions of CB2-Rs significantly increased in PFC, Hip, NAc and VTA of $2 \mathrm{~Hz}$ EA treated rats compared with heroin SA rats.

\section{Conclusions}

Our present study adopted the heroin SA model rats to explore whether $2 \mathrm{~Hz}$ EA inhibited the heroinevoked seeking behaviors and regulated the expression of CB1-Rs and CB2-Rs in mesolimbic brain. The results showed that $2 \mathrm{~Hz}$ EA could attenuate the heroin evoked seeking and relapse behaviors. The inhibitory effect of $2 \mathrm{~Hz}$ EA might be related to down-regulate the expression of CB1-Rs and up-regulation the expressions of CB2-Rs in relapse-relevant brain regions in heroin-addicted rats. The CB1-Rs and CB2-Rs in mesolimbic brain were potential targets for $2 \mathrm{~Hz}$ EA treatment of drug addiction and relapse.

\section{Conflict of Interest}

There is no conflict of interest.

\section{Acknowledgements}

This study was supported by the National Natural Science Foundation of China (No. 81473442 to L. Chen, No. 31600968 to Y.-X. Wu), Wuhan Science and Technology Department (Grant No. 2017060201010166).

\section{References}

BAYEWITCH M, AVIDOR-REISS T, LEVY R, BARG J, MECHOULAM R, VOGEL Z: The peripheral cannabinoid receptor: adenylate cyclase inhibition and G protein coupling. FEBS Lett 375: 143-147, 1995.

CHEN DJ, GAO M, GAO FF, SU QX, WU J: Brain cannabinoid receptor 2: expression, function and modulation. Acta Pharmacol Sin 38: 312-316, 2017.

CHEN LMB, YUE K, RU Q, TIAN X, XIONG Q, LIU L, LI CY: 2Hz-Electroacupuncture attenuates conditioned cue-evoked heroin-seeking behavior and increase CB2-Rs expression in relapse-relevant brain regions in heroin-addicted rats. Adv Mater Res 998-999: 164, 2014. 
CHEN L, ZHANG J, LI F, QIU Y, WANG L, LI YH, SHI J, PAN HL, LI M: Endogenous anandamide and cannabinoid receptor-2 contribute to electroacupuncture analgesia in rats. J Pain 10: 732-739, 2009.

CHENG XR, YANG Y, ZHOU WX, ZHANG YX:; Expression of VGLUTs contributes to degeneration and acquisition of learning and memory. Neurobiol Learn Mem 95: 361-375, 2011.

CUI CL, WU LZ, LI YJ: Acupuncture for the treatment of drug addiction. Int Rev Neurobiol 111: 235-256, 2013.

DA SILVA AN: Acupuncture for migraine prevention. Headache 55: 470-473, 2015.

DONG Y, TAYLOR JR, WOLF ME, SHAHAM Y: Circuit and synaptic plasticity mechanisms of drug relapse. J Neurosci 37: 10867-10876, 2017.

GARCIA PARDO MP, ROGER SANCHEZ C, DE LA RUBIA ORTI JE, AGUILAR CALPE MA: Animal models of drug addiction. Adicciones 29: 278-292, 2017.

GARDNER EL: Endocannabinoid signaling system and brain reward: emphasis on dopamine. Pharmacol Biochem Behav 81: 263-284, 2005.

GELBIER S: The origins and nature of acupuncture in dentistry. Dent Hist 61: 5-9, 2016.

HAN JS: Acupuncture and endorphins. Neurosci Lett 361: 258-261, 2004.

HAN JS, ZHANG RL: Suppression of morphine abstinence syndrome by body electroacupuncture of different frequencies in rats. Drug Alcohol Depend 31: 169-175, 1993.

HU B, BAI F, XIONG L, WANG Q: The endocannabinoid system, a novel and key participant in acupuncture's multiple beneficial effects. Neurosci Biobehav Rev 77: 340-357, 2017.

KIM JH, CHUNG JY, KWON YK, KIM KJ, YANG CH, HAHM DH, LEE HJ, PYUN KH, SHIM I: Acupuncture reduces alcohol withdrawal syndrome and c-Fos expression in rat brain. Am J Chin Med 33: 887-896, 2005.

KIM MR, KIM SJ, LYU YS, KIM SH, LEE Y, KIM TH, SHIM I, ZHAO R, GOLDEN GT, YANG CH: Effect of acupuncture on behavioral hyperactivity and dopamine release in the nucleus accumbens in rats sensitized to morphine. Neurosci Lett 387: 17-21, 2005.

KOOB GF, MALDONADO R, STINUS L: Neural substrates of opiate withdrawal. Trends Neurosci 15: 186-191, 1992. KOSTEN TR, O'CONNOR PG: Management of drug and alcohol withdrawal. $N$ Engl J Med 348: 1786-1795, 2003.

LIANG J, PING XJ, LI YJ, MA YY, WU LZ, HAN JS, CUI CL: Morphine-induced conditioned place preference in rats is inhibited by electroacupuncture at $2 \mathrm{~Hz}$ : role of enkephalin in the nucleus accumbens. Neuropharmacology 58: 233-240, 2010.

LIU S, ZHU F, LAI M, SUN L, LIU Y, ZHOU W: Electroacupuncture suppresses discrete cue-evoked heroin-seeking and fos protein expression in the nucleus accumbens core in rats. Evid Based Complement Alternat Med 2012: 286404, 2012.

MEAD AN: Appropriate experimental approaches for predicting abuse potential and addictive qualities in preclinical drug discovery. Expert Opin Drug Discov 9: 1281-1291, 2014.

MITRIRATTANAKUL S, LOPEZ-VALDES HE, LIANG J, MATSUKA Y, MACKIE K, FAULL KF, SPIGELMAN I: Bidirectional alterations of hippocampal cannabinoid 1 receptors and their endogenous ligands in a rat model of alcohol withdrawal and dependence. Alcohol Clin Exp Res 31: 855-867, 2007.

MORALES M, BONCI A: Getting to the core of addiction: hooking CB2 receptor into drug abuse? Nat Med 18: 504-505, 2012

NESTLER EJ: Molecular mechanisms of drug addiction. Neuropharmacology 47 (Suppl 1): 24-32, 2004.

ONAIVI ES: Neuropsychobiological evidence for the functional presence and expression of cannabinoid CB2 receptors in the brain. Neuropsychobiology 54: 231-246, 2006.

ONAIVI ES: Commentary: functional neuronal CB2 cannabinoid receptors in the CNS. Curr Neuropharmacol 9: 205-208, 2011.

PANAGIS G, MACKEY B, VLACHOU S: Cannabinoid regulation of brain reward processing with an emphasis on the role of cb1 receptors: a step back into the future. Front Psychiatry 5: 92, 2014.

PARSONS LH, HURD YL: Endocannabinoid signalling in reward and addiction. Nat Rev Neurosci 16: 579-594, 2015.

SAGHEDDU C, MUNTONI AL, PISTIS M, MELIS M: Endocannabinoid signaling in motivation, reward, and addiction: influences on mesocorticolimbic dopamine function. Int Rev Neurobiol 125: 257-302, 2015.

SERRANO A, PARSONS LH: Endocannabinoid influence in drug reinforcement, dependence and addiction-related behaviors. Pharmacol Ther 132: 215-241, 2011. 
SHEN F, WANG N, QI C, LI YJ, CUI CL: The NO/sGC/PKG signaling pathway in the NAc shell is necessary for the acquisition of morphine-induced place preference. Behav Neurosci 128: 446-459, 2014.

SHI XD, REN W, WANG GB, LUO F, HAN JS, CUI CL: Brain opioid-receptors are involved in mediating peripheral electric stimulation-induced inhibition of morphine conditioned place preference in rats. Brain Res 981: 23-29, 2003.

TANG YL, HAO W: Improving drug addiction treatment in China. Addiction 102: 1057-1063, 2007.

TSEUNG YK: Letter: Acupuncture for drug addiction. Lancet 2: 839, 1974.

WANG X, LUO YX, HE YY, LI FQ, SHI H S, XUE LF, XUE YX, LU L: Nucleus accumbens core mammalian target of rapamycin signaling pathway is critical for cue-induced reinstatement of cocaine seeking in rats. $J$ Neurosci 30: $12632-12641,2010$.

WILLS KL, PARKER LA: Effect of pharmacological modulation of the endocannabinoid system on opiate withdrawal: a review of the preclinical animal literature. Front Pharmacol 7: 187, 2016.

WU LZ, CUI CL, TIAN JB, JI D, HAN JS: Suppression of morphine withdrawal by electroacupuncture in rats: dynorphin and kappa-opioid receptor implicated. Brain Res 851: 290-296, 1999.

XI ZX, PENG XQ, LI X, SONG R, ZHANG HY, LIU QR, YANG HJ, BI GH, LI J, GARDNER EL: Brain cannabinoid $\mathrm{CB}(2)$ receptors modulate cocaine's actions in mice. Nat Neurosci 14: 1160-1166, 2011.

YUE K, MA B, RU Q, CHEN L, GAN Y, WANG D, JIN G, LI C: The dopamine receptor antagonist levotetrahydropalmatine attenuates heroin self-administration and heroin-induced reinstatement in rats. Pharmacol Biochem Behav 102: 1-5, 2012.

ZACHARIOU V, BOLANOS CA, SELLEY DE, THEOBALD D, CASSIDY MP, KELZ MB, SHAW-LUTCHMAN T, BERTON O, SIM-SELLEY LJ, DILEONE RJ, KUMAR A, NESTLER EJ: An essential role for DeltaFosB in the nucleus accumbens in morphine action. Nat Neurosci 9: 205-211, 2006.

ZHANG F: SuperState: a computer program for the control of operant behavioral experimentation. J Neurosci Methods 155: 194-201, 2006.

ZHANG J, CHEN L, SU T, CAO F, MENG X, PEI L, SHI J, PAN HL, LI M: Electroacupuncture increases $\mathrm{CB} 2$ receptor expression on keratinocytes and infiltrating inflammatory cells in inflamed skin tissues of rats. J Pain 11: 1250-1258, 2010.

ZHAO ZQ: Neural mechanism underlying acupuncture analgesia. Prog Neurobiol 85: 355-375, 2008. 